\title{
«LA FISIOLOGÍA INVISIBLE»: recursos audiovisuales para la enseñanza de la fisiología en ciencias de la salud
}

Carlos Romá-Mateo $^{\text {a }}$, Conrado J. Calvo ${ }^{\text {, Gloria Olaso-González }}{ }^{\mathrm{c}}$

Departamento de Fisiología, Facultad de Medicina y Odontología. Universitat de València. acarlos.roma@uv.es; b conrado.calvo@uv.es; ${ }^{\mathrm{a}}$ gloria.olaso@uv.es

\begin{abstract}
Human physiology is a basic subject during the first courses related to health sciences. Physiology as a discipline is markedly heterogeneous, since it includes anatomical, functional, molecular and biochemical notions, all widely interconnected. For this reason, it is difficult for students to integrate this knowledge, especially in the first years where the rest of related subjects (general biology, biochemistry, etc.) are taken at the same time. To facilitate this learning, the audio-visual material implies a great support when it comes to specifying abstract concepts and data obtained through complex experiments. Currently students are digital natives, and find the audiovisual tools intuitive, facilitating their learning. After using resources of marked visual character (videos and online comics) in General Physiology classes in the Medicine degree, and based on the students' responses to specific surveys on the usefulness of said resources, we have designed a plan for the creation of a series of videos, which could contribute to student motivation, renew the teaching methodology, and also be used as free access content for other subjects and degrees, contributing at the same time to the promotion of 'open' scientific knowledge in society.
\end{abstract}

Keywords: methodologies, ICT, audiovisual, physiology, health, science

\section{Resumen}

La fisiología humana es una asignatura básica en los primeros cursos relacionados con las ciencias de la salud. La fisiología como disciplina es marcadamente heterogénea, pues comprende nociones anatómicas, funcionales, moleculares y bioquímicas, todas ampliamente interconectadas. Por este motivo, es dificil para los estudiantes integrar este conocimiento, especialmente en los primeros años donde el resto de asignaturas de apoyo (biología general, bioquímica, etc) se cursan al mismo tiempo. Para facilitar este aprendizaje, el material audiovisual supone un gran apoyo a la hora de concretar conceptos abstractos y datos obtenidos mediante complejos experimentos. Actualmente los alumnos son nativos digitales, y las herramientas audiovisuales son intuitivas, facilitadoras de su aprendizaje. Tras utilizar recursos de marcado carácter visual (videos y cómics en línea) en clases de Fisiología General en grado de Medicina, y a partir de las respuestas de los alumnos a encuestas especificas sobre la utilidad de dichos 
recursos, hemos diseñado un plan de realización de una serie de videos, que puedan contribuir a la motivación del estudiante, renovar la metodología docente, y además ser utilizados como contenido de libre acceso para otras asignaturas y titulaciones, contribuyendo al mismo tiempo a la promoción del conocimiento cientifico 'en abierto' en la sociedad.

Palabras clave: metodologías, TIC, audiovisual, fisiología, salud, ciencia

\section{Introducción}

La fisiología humana es una asignatura clave en la mayoría de grados en el ámbito de ciencias de la salud, teniendo una carga docente especialmente elevada en grados como el de medicina. Sin embargo, la comprensión de la fisiología general incluso en los niveles más iniciales de la carrera universitaria requiere de una robusta base en torno a materias como bioquímica, biología molecular y biología celular que a menudo excede lo impartido en la Educación Secundaria Obligatoria y el Bachillerato. Además, se da el caso de estudiantes que provienen de ramas de secundaria en las que las asignaturas de ciencia son minoritarias. De este modo, los profesores encuentran importantes lagunas en conceptos básicos como puedan ser el de $\mathrm{pH}$, las reacciones de óxido-reducción, nociones básicas de metabolismo o la estructura celular. Si bien estos conceptos se abordan en otras asignaturas como Bioquímica General o Biología, la cronicidad de las asignaturas a veces no facilita la integración de dichos conceptos en el momento adecuado. Por lo tanto, el profesor de fisiología debe echar mano de recursos alternativos y complementarios para poder introducir el temario y reforzar los temas, dentro del ajustado calendario académico. Además de este problema, se da la circunstancia de que las nociones de biología molecular suponen para el estudiantado un abstracción difícil de visualizar; la propia falta de familiaridad con la naturaleza y estructura de las macromoléculas biológicas o con la química orgánica convierten los esquemas y fórmulas utilizados por los profesores en crípticos jeroglíficos que los estudiantes, finalmente, optan por memorizar más que por razonar. En la clase hay poco tiempo para ofrecer además de los esquemas y apoyo gráfico necesarios, las claves para descifrarlos, por lo que el uso de material complementario de referencia se muestra crucial. En particular, el profesor debe evitar la aprehensión por parte del alumnado de los mecanismos fisiológicos y su regulación de forma automatizada y mecánica, sin cuestionar la base celular y molecular que los origina, la cual a menudo permanece "invisible" frente a las consecuencias anatómico-patológicas que en la práctica terminan por memorizar.

Las nuevas tecnologías han cambiado radicalmente la manera de abordar las clases. Las Tecnologías de la Información y la Comunicación (TIC) han supuesto un re-enfoque de las clases a todos los niveles, tanto por parte del profesor en lo tocante a documentación, material de apoyo y tipo de actividades formativas, como por parte del alumnado, que no solo las utiliza en las horas de trabajo autónomo, sino que sigue las clases utilizando dispositivos informáticos con conexión a internet. A fecha de hoy, conseguir mantener la atención de los alumnos pasa por aprovechar esta circunstancia, proporcionando material de apoyo visual que permita seguir fácilmente las explicaciones del profesor y estimule la capacidad 
razonadora del estudiante a la hora de interpretar metáforas y analogías que representan una realidad compleja. Investigaciones en los últimos años han demostrado que el uso de videos y animaciones son herramientas pedagógicas capaces de potenciar el proceso de aprendizaje. (Dong 2015) (Brame 2018). Estudios aislados han demostrado que la percepción del uso de videos por parte de los alumnos es una clara mejora en su aprendizaje y comprensión de conceptos complejos, así como una mejora hacia sus estrategias de aprendizaje personal (Boateng 2016). Del mismo modo el empleo de estrategias de refuerzo mixtas han mostrado ser de interés para promover el aprendizaje autónomo (Mahaffey 2018). El uso de videos en la formación en salud ha demostrado apoyar y promover el aprendizaje ofreciendo grandes ventajas respecto a metodologías tradicionales, como la adquisición autónoma de competencias específicas y transversales; sin embargo, la elección de los materiales adecuados y efectivos sigue siendo un desafío para el profesor universitario (Hurtubise 2013).

La profusión de materiales en línea ya preparados por terceros y en especial la creciente oleada de material de divulgación científica de gran calidad permite echar mano de recursos llamativos y a la vez rigurosos. Nuestra experiencia previa con el uso de videos, aplicaciones informáticas o incluso cómics de divulgación científica en el aula ha mostrado que los alumnos acogen positivamente este tipo de materiales, y que en ocasiones estimula su capacidad reflexiva, sus competencias de interpretación de la información y en términos generales su capacidad de aprendizaje. En años anteriores, la utilización de los videos de la serie "Crash Course" creados por John Green y Hank Green, tanto en la asignatura de Fisiología General en Medicina como la asignatura de Morfología y Función II, de los complementos formativos del Máster Universitario en Ingeniería Biomédica, demostró que los videos online de una duración breve (entre 3 y 10 minutos máximo) y con un tono tan desenfadado como riguroso, relajan el ambiente de clase, pero además preparan al estudiante para el abordaje de temas complejos y a menudo áridos. Igualmente bien recibida ha sido la utilización de algunas páginas del cómic The OOBIK proteo-type, creado por el investigador principal en colaboración con el ilustrador Gerardo Sanz, para romper la aridez de los esquemas típicos de libros de texto y promoviendo el ejercicio interpretativo y de descodificación de la información, competencias características de la comunicación científica y tan necesarias en el dominio de la fisiología humana.

El presente trabajo nace a raíz de un proyecto de innovación docente aprobado por la CAT de la Facultad de Medicina y Odontología de la Universitat de València y financiado a través de la convocatoria de renovación de metodologías docentes de esta misma universidad (UVSFPIE_RMD18-841877), con la intención de explotar el potencial real de este tipo de materiales educativos mediante una estrategia dividida en dos fases: primero, evaluando y cuantificando la percepción de los alumnos hacia el uso de materiales ya existentes, al tiempo que se detecta las unidades temáticas donde su aplicación pueda ser más eficiente; por otro lado, en vistas a desarrollar materiales optimizados propios que cubran específicamente las necesidades detectadas. 


\section{Objetivos}

El objetivo principal del proyecto es la evaluación de la utilidad de materiales y recursos gráficos y audiovisuales en-línea para la enseñanza de la fisiología en asignaturas de ciencias de la salud. Este objetivo más general puede a su vez definirse en base a los siguientes objetivos específicos:

- Utilizar material en línea audiovisual (videos, animaciones, cómics) en las clases de teoría de la asignatura de Fisiología General.

- Detectar y evaluar el impacto de dichos materiales a través de cuestionarios en línea, así como identificar los temas y conceptos más necesitados de un refuerzo complementario en el que dicho material pueda ser especialmente útil, dentro del temario de Fisiología General.

- Plantear buenas prácticas para la elaboración de una serie de materiales docentes en formato video de corta duración, que suplan las carencias detectadas y sirvan para estimular el seguimiento de la asignatura y la participación del alumnado.

- Evaluar la utilidad de los videos elaborados y ensayar su utilización en otras asignaturas afines.

\section{Desarrollo de la innovación}

\subsection{Características del curso}

El trabajo se ha realizado a lo largo de la impartición de la asignatura cuatrimestral Fisiología General, de primer curso del Grado en Medicina de la Universitat de València, y en particular en el grupo ARA, donde la docencia se realiza en lengua inglesa. Las clases consisten mayoritariamente en el formato de clases magistrales, pero dirigidas en gran medida por preguntas y respuestas entre profesor y alumnos, y entre los cuatro bloques temáticos que componen el temario se realizan sesiones de aclaración de dudas y conceptos en los que se utiliza como actividad dinamizadora y de chequeo de conocimientos un test mediante la herramienta de votación electrónica en-línea Kahoot.

\subsection{Material utilizado}

\subsubsection{Videos}

Los videos utilizados han sido elegidos por los miembros del equipo por su capacidad para sintetizar de manera gráfica y efectiva conceptos complejos, su narrativa para captar el interés de los alumnos y plantear cuestiones de interés, ser recursos en abierto, y en función de las particularidades de cada tema. Los videos de la serie Crash Course (Crash course biologyCrash Course physiology) han sido elegidos como apoyo tanto de la introducción al tema, como en el punto de la clase que requiriese una aclaración específica contenida en el video. 
Adicionalmente, se ha propuesto el visionado de algunos de estos videos con conceptos avanzados y de refuerzo como trabajo autónomo por parte del estudiante (Mahaffey 2018).

\subsubsection{Cómics}

A lo largo del curso se ha utilizado extractos del cómic en-línea The OOBIK proteo-type para plantear actividades de trabajo autónomo, durante las lecciones y a través del Aula Virtual de la asignatura (Moodle). Se trata de actividades únicamente de evaluación formativa, no cuantitativa, que no puntúan sobre la nota final del curso y de carácter voluntario. Dado que el cómic es creación del autor principal del trabajo, además se presta su utilización para formar parte de los videos que se realizarán a partir de los resultados de las encuestas.

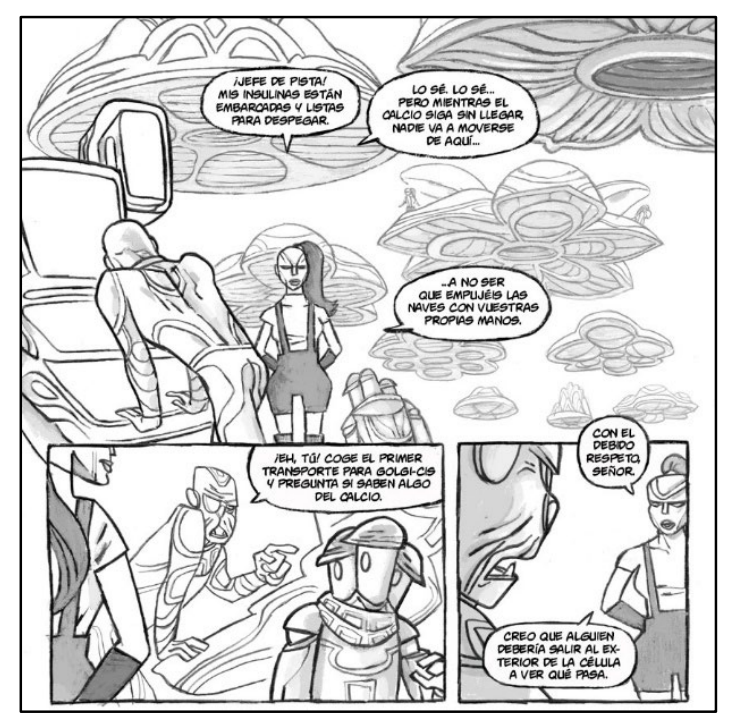

Fig. 1 Detalle de página del cómic en-línea The OOBIK proteo-type utilizada en un ejercicio sobre exocitosis a través del aula virtual del curso

\subsection{Encuestas}

Las encuestas se han realizado de forma anónima a través del Aula Virtual. Incluyen un bloque de caracterización de los estudiantes (datos biográficos), mediante respuestas de opción restringida o abierta, según el caso; un bloque enfocado a conocer la opinión de los estudiantes en cuanto a la metodología docente de la asignatura; un tercer bloque para conocer las características más valoradas en los videos como complemento de asignatura; y un bloque para conocer la influencia de este tipo de iniciativas en sus resultados de aprendizaje. Todas estas preguntas fueron del tipo de valoración mediante escala graduada de 1 (total desacuerdo) a 5 (completamente de acuerdo) de una serie de afirmaciones. Finalmente, se incluye un espacio de texto libre donde además de recabar opiniones y sugerencias personales, se insta a indicar le tipo de material audiovisual preferido a lo largo del curso, los temas más idóneos para incorporar material audiovisual, etc. 


\section{Resultados}

En el momento de la presente comunicación, y una vez terminada la impartición de la asignatura, se está realizando el primer video de la serie en base a las directrices derivadas de las encuestas realizadas a los alumnos.

\subsection{Encuestas preliminares}

De los 76 alumnos matriculados en la asignatura, 38 respondieron a las encuestas. En la Fig.2 se resumen los datos demográficos: la gran mayoría de estudiantes son de nacionalidad española y tienen 18 años, siendo la proporción de géneros de aproximadamente dos tercios femenino sobre un tercio masculino. El total de los estudiantes encuestados declaró estar matriculado por primera vez en el curso. Estos datos revelan la muestra de estudiantes utilizada como bastante homógenea, si bien podría resultar interesante en una futura ampliación del estudio, analizar las diferencias en percepción de la utilidad de los videos en función del género.
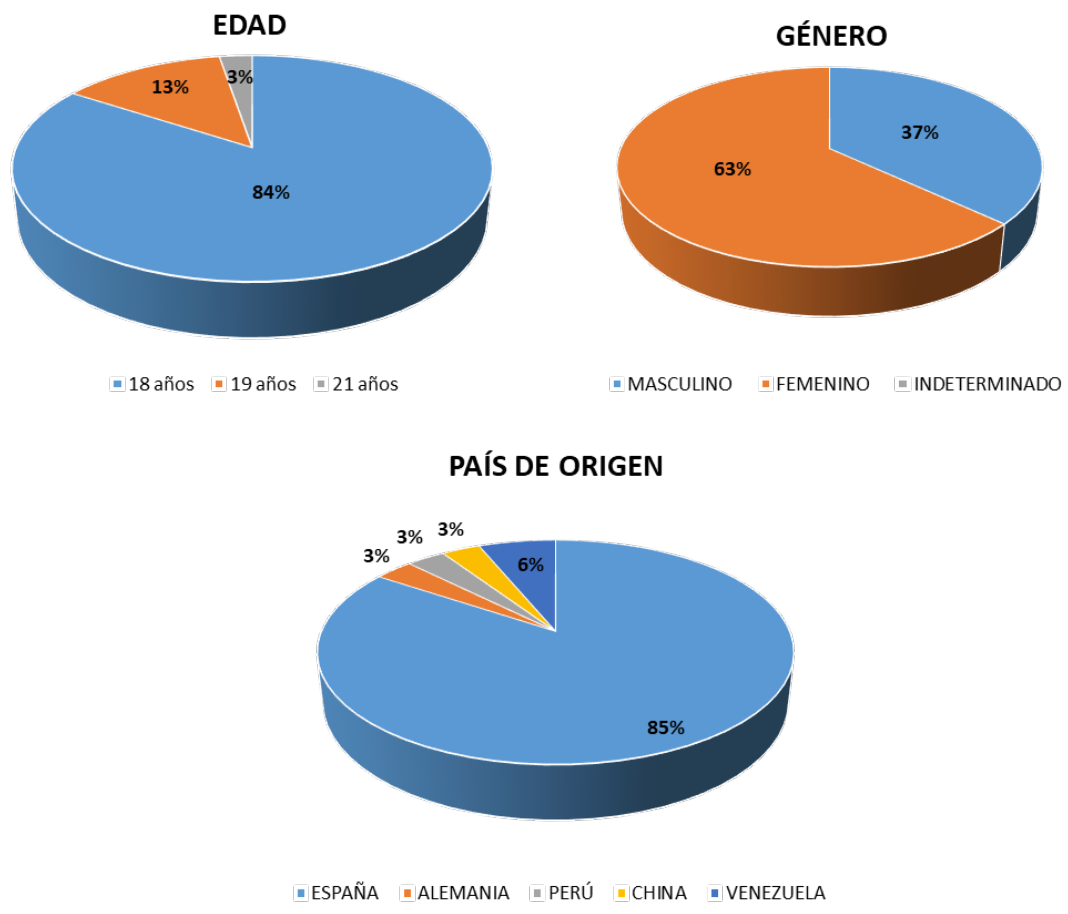

Fig. 2 Perfil de los estudiantes encuestados $(n=38)$

\subsubsection{Metodología general de la asignatura}


Respecto a la metodología docente en términos de utilización de recursos, la mayoría de estudiantes considera que los materiales subidos por el profesor al aula virtual son útiles para seguir las clases (4’3 en promedio), y en concreto se valora positivamente la uilización de recursos no estrictamene académicos (4’2). Respecto a la naturaleza de dichos recursos, la utilización de videos y animaciones obiene un $4^{\prime} 8$, seguido de infografías gráficas con un 4’2. Un resultado interesante es la baja valoración de páginas de cómic o webcomics, que obtiene un 3'7 (Fig. 3).

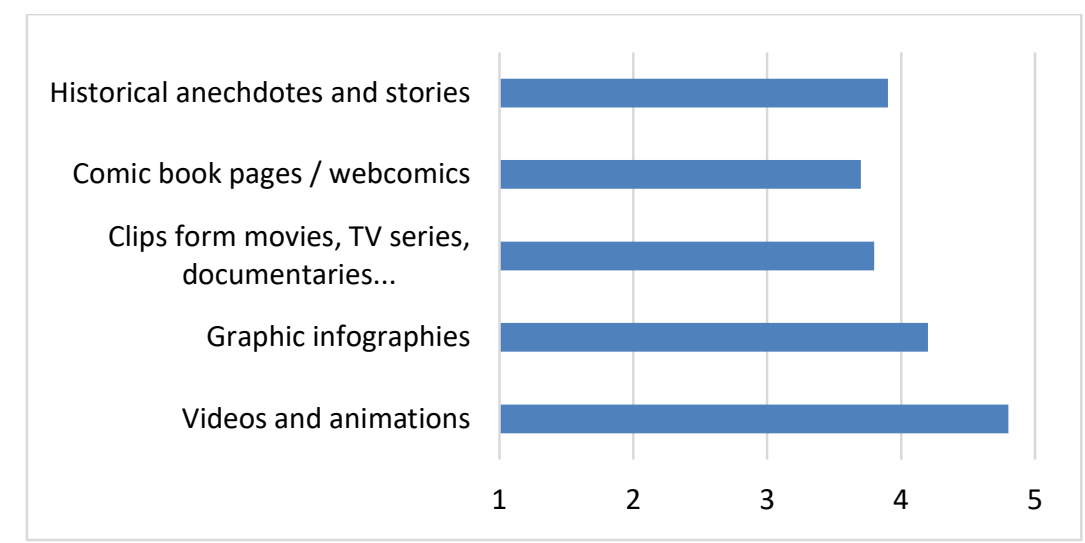

Fig. 3 Promedio de puntuaciones obtenidas (1-5) sobre la conformidad de los estudiantes en cuanto a afirmaciones referentes a la utilidad de diversos recursos utilizados durante las clases

\subsubsection{Características de los videos}

Al ser preguntados acerca de las características más deseables para los videos, obtenemos algunos resultados interesantes. En primer lugar, en cuanto a la distribución temporal de los videos, la mayor puntuación se obtiene para las opciones que implican el visionado de los videos durante la clase; en primer lugar gana la opción de insertar el video en el momento concreto en que una explicación de un concepto complejo o abstracto lo requiere (4'4) (Fig. 4). Más interesante todavía resulta el hecho de que se obtiene muy baja puntuación para la opción de ver el video en casa a modo de trabajo autónomo para reforzar una clase y enlazar con concpetos de la siguiene (3'8), pregunta encaminada a dirigir uno de los principales objetivos de nuestro proyecto basado en reforzar la metodología de aula invertida. 
The video is watched at home (autonomous work for the student), after reviewing the lecture and in order to expand or clarify...

The video is shown after the class, as a summary/recap

The video is shown during the class, explaining a concept difficult to imagine or visualize

The video is shown in class, introducing the lecture

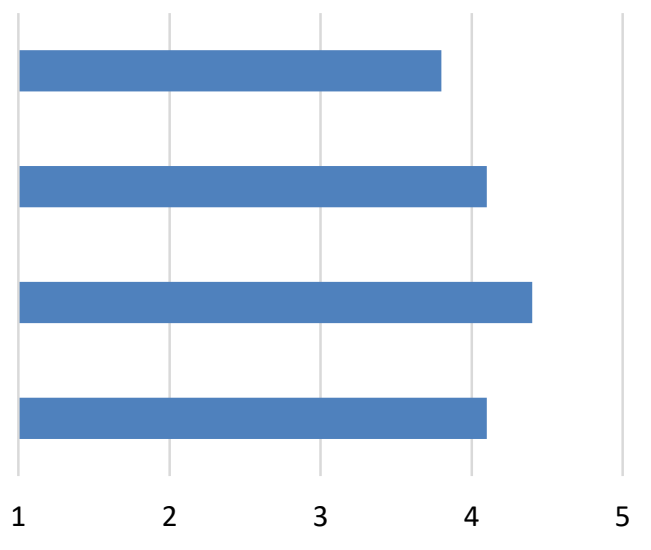

Fig. 4 Promedio de puntuaciones obtenidas (1-5) sobre el momento preferido por los estudiantes para visualizar videos relacionados con la asignatura

En cuanto a los aspectos formales de los videos, los estudiantes mostraron preferencia por el hecho de que el video incluyese un narrador (4'5), que sirviese para responder preguntas específicamente planteadas durante las clases (4’2) y que pudiera servir de enlace entre dos clases distintas (4’0). Entre lo menos valorado, que el video fuese presentado por el mismo profesor de la asignatura $\left(3^{\prime} 0 / 5\right)$ y, de forma interesante, también se valoró menos el hecho de que el video incluyese referencias científicas actuales en el campo de estudio específico de la asignatura (3’4) (Fig. 5).

\section{The video serves as a link between two} different lectures

The video answers specific questions raised by the teacher during the lectures

The video includes jokes and references to popular culture

The video involves references to the most actual scientific research in the particular field

The video explains details or information beyond concepts seen in the lecture

The person presenting the video is your physiology professor

The video includes a speaker (narrative either in first/third person)

The video shows animated writing/drawing in a screen

The video is completely made of animations/cartoons

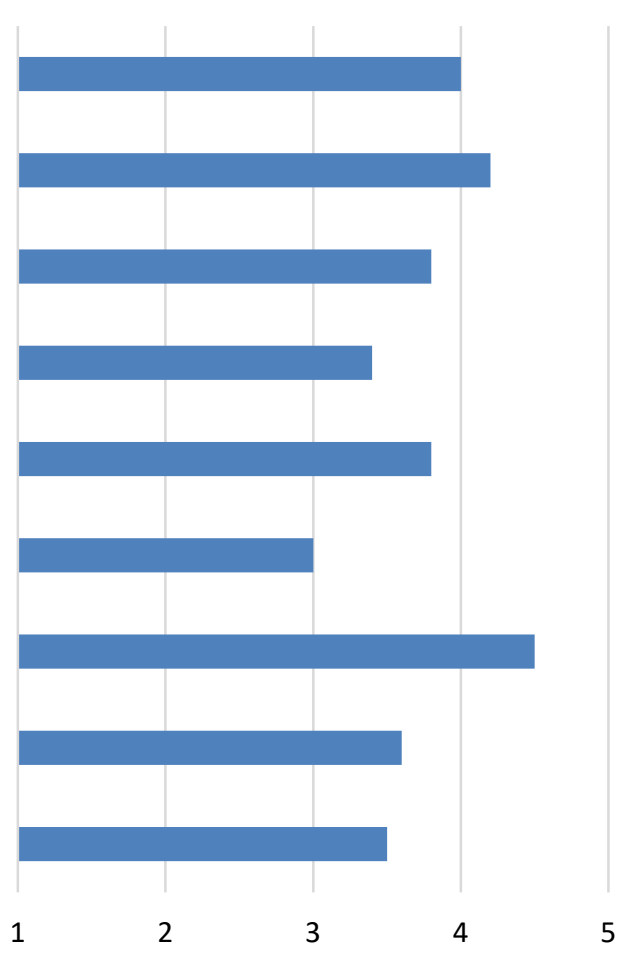

5

Fig. 5 Promedio de puntuaciones obtenidas (1-5) respecto a las características formales de los videos 


\subsubsection{Valoración de la experiencia personal}

Al ser preguntados por su impresión acerca de cómo los videos contribuyen a sus resultados de aprendizaje, los estudiantes manifiestan que estos ayudan en la comprensión de cuestiones técnicas complejas y difíciles de visualizar (4'5), que facilitan el desarrollo de pensamiento crítico y desarrollo de resolución de problemas (4’5), y estimulan su atención durante la clase incrementando su motivación para aprender los conceptos de la asignatura (4'4). De nuevo destaca la clara preferencia por el uso de los videos como complemento de las lecciones (4'1) frente a la posibilidad de trabajar con los videos en casa, de manera complementaria al resto de recursos educativos ( $\left.3^{\prime} 4\right)$.

\subsubsection{Comentarios y sugerencias}

De entre los 38 estudiantes que respondieron a la encuesta, 34 dejaron comentarios en el espacio dedicado al texto libre para sugerencias y aclaraciones. En la Tabla 1 se ofrece un resumen de algunos de los comentarios destacados.

Tabla 1. Extracto de algunos ejemplos de comentarios y sugerencias realizados por los estudiantes

\begin{tabular}{|c|c|}
\hline $\begin{array}{l}\text { OBJETO DEL } \\
\text { COMENTARIO }\end{array}$ & CITA TEXTUAL \\
\hline $\begin{array}{l}\text { Sobre el uso del } \\
\text { cómic en las clases }\end{array}$ & $\begin{array}{l}\text { I have read the comic Carlos Romá is doing with one friend and I find it really } \\
\text { interesting and useful, since it helped me to understand some concepts I didn't have } \\
\text { very clear and it's an easy way to learn and don't forget them. }\end{array}$ \\
\hline \multirow{6}{*}{$\begin{array}{l}\text { Sobre el uso de } \\
\text { videos en clase }\end{array}$} & $\begin{array}{l}\text { From my point of view, the videos from the crash course were very useful and graphic } \\
\text { in order to understand the general concepts and to imagine them. }\end{array}$ \\
\hline & $\begin{array}{c}\text { I think the crash course videos are really helpful, as well as those with someone } \\
\text { drawing, but these are better to see at home, in order to review what we have seen in } \\
\text { class. }\end{array}$ \\
\hline & My favourite type of videous are the ones that crash course make, they are $100 \%$ useful \\
\hline & $\begin{array}{l}\text { I am really happy about the videos and animations suggested and showed in class. } \\
\text { They've helped me in understanding some complex processes mainly the ones about } \\
\text { channels }\end{array}$ \\
\hline & $\begin{array}{l}\text { I think the crash course videos were very useful specially to understand the general } \\
\text { concepts }\end{array}$ \\
\hline & $\begin{array}{c}\text { "Crash Course" or "Kurzgesagt - In a Nutshell" are a good example of entertaining and } \\
\text { educational videos. }\end{array}$ \\
\hline
\end{tabular}




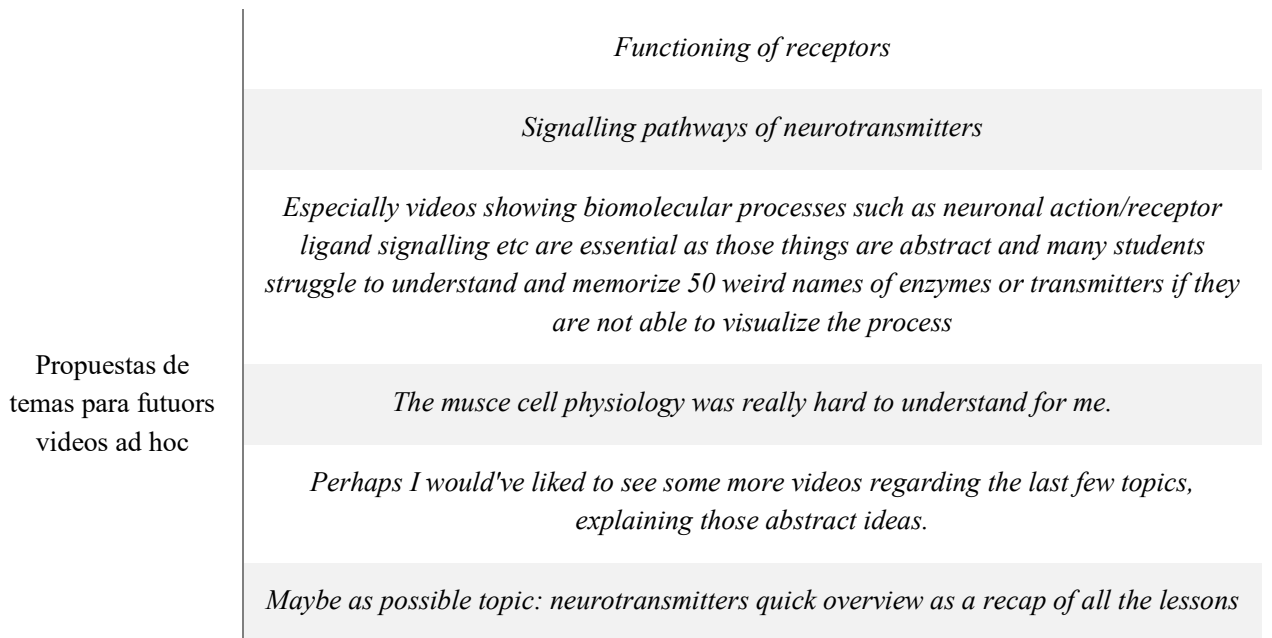

En general, los comentarios son favorables en cuanto a la utilización de videos durante las clases; de todos los videos utilizados, la serie Crash Course se menciona como preferente hasta en 12 ocasiones distintas. En cuanto a los temas sugeridos como temas idóneos para ser representados en forma de videos, lo más mencionado es el tema de la señalización intracelular (receptores, segundos mensajeros, etc) seguido del tema de los diferentes tipo de neurotransmisores y de la fisiología muscular. En cuanto al tono y profundidad de la temática de los videos, existe heterogeneidad: en general la mayoría de estudiantes agradecen un tono desenfadado y la presencia de chistes o anécdotas divertidas en los videos, mientras que unos pocos se decantan por un estilo más serio y académico.

En su conjunto, las respuestas a las preguntas de los anteriores bloques, y las impresiones reflejadas en este último bloque de comentarios, conforman una muy útil perspectiva de la visión del estudiante sobre la utilidad de los videos como complemento a la enseñanza de la fisiología. Pero además, permite establecer una serie de características preferentes a la hora de diseñar ad hoc material audiovisual adecuado para ser utilizado tanto en esta asignatura como en otras de carácter similar centradas en la fisiología humana. 


\section{Conclusiones}

En base a los resultados obtenidos, los criterios para diseñar el video piloto a efectos de ser testado en asignaturas de físiología serán:

a. Utilizando como modelo los videos de la serie Crash Course, los videos estarán presentados por el profesor de la asignatura

b. A diferencia de los primeros, la presencia del profesor será testimonial, presentando el video, los conceptos que se desarrollarán o explicarán a lo largo del mismo, y en el caso pertinente retomando alguna de las preguntas planteadas durante las clases

c. El tema del primer video será una introducción al nivel molecular de la fisiología humana, la parte más "invisible" del temario, y centrado especialmente en las rutas de señalización intracelulares.

d. El video incluirá esquemas, dibujos, y animaciones así como recursos gráficos extraídos del cómic The OOBIK proteo-type.

La grabación se realizará en los estudios del Servei de Formació Permanent i Innovació Educativa de la Universitat de València, donde se dispone de todo de tipo de recursos audiovisuales para cumplir con estos requisitos. Por lo tanto, el presente trabajo supone la base para cimentar un proyecto a largo plazo, donde en sucesivos cursos se ponga en práctica y se evalúe la eficiencia de estas nuevas herramientas y se continúe ampliando la colección al tiempo que se diversifica y se aplica en otras asignaturas donde la fisiología tenga también un peso importante. El objetivo final será la tendencia hacia un estilo de impartición de la fisiología general donde se combine la estrategia del aula invertida y la clase magistral tradicional, facilitado por la disponibilidad de los videos y la posibilidad de plantear en ellos cuestiones que potencien el trabajo autónomo y sirvan para preparar las sucesivas clases por parte de los alumnos. No obstante, los resultados obtenidos apuntan a una falta de motivación por parte del alumnado hacia la metodología de aula invertida, lo cual se tendrá muy en cuenta a la hora tanto de diseñar nuevos recursos, como de evaluar su impacto real sobre la motivación y, en especial, los resultados de aprendizaje del alumnado.

\section{Referencias}

BOATING R., et al (2016). "Videos in learning in higher education: assessing perceptions and attitudes of students at the University of Ghana" en Smart Learning Environments 3:8

BRAME C.J. et al. (2018). "Effective Educational Videos (Online)". <https://cft.vanderbilt. edu/guides-sub-pages/effective-educational-videos/> [Consulta: 3 mayo 2018].

DONG C. y GOH P.S. (2015). "Twelve tips for the effective use of videos in medical education" en Med Teach vol. 37, p. 140-145.

GREEN J. y GREEN H. Crashcourse

$<$ https://www.youtube.com/user/crashcourse/featured $>$ [Consulta: 15 septiembre 2018] 
«LA FISIOLOGÍA INVISIBLE»: recursos audiovisuales para la enseñanza de la fisiología en ciencias de la salud

HURTUBISE L., MARTIN B., et al. (2013). "To play or not to play: Leveraging video in medical education" en Journal of Graduate Medical Education vol. 5, issue 1, p. 13-18

MAHAFFEY A.L., et al. (2018). "Interfacing virtual and face-to-face teaching methods in an undergraduate human physiology course for health professional students" en Adv. Physiol Ed. Vol. 42 p. $477-481$

ROMÁ-MATEO C. y SANZ G., en PRINCIPIA. The OOBIK proteo-type < $<$ ttps://principia.io/theoobik/> [Consulta: 4 diciembre 2018 ] 